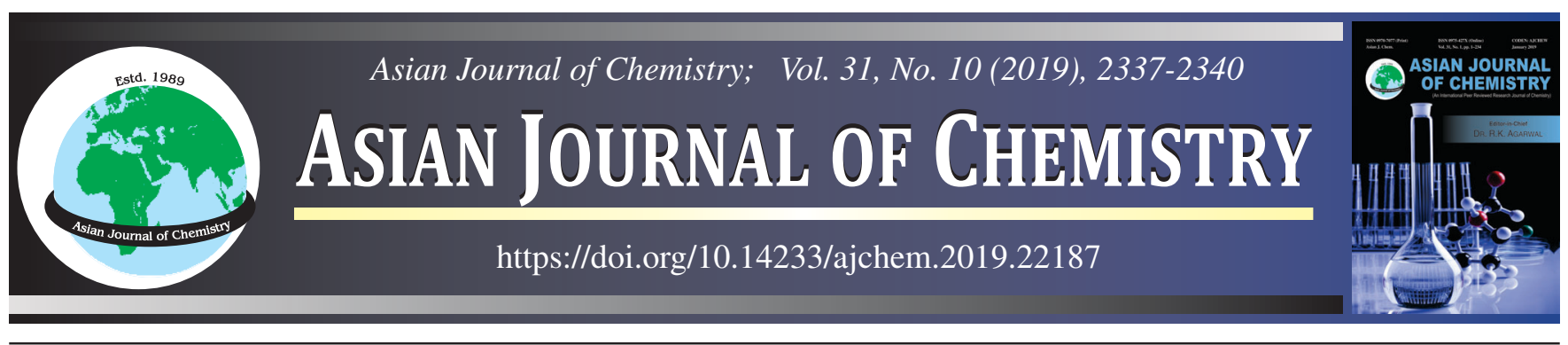

\title{
Synthesis and Spectral Characterization of D-A Based Cyano-Stilbene Derivatives for Organic Solar Cell Applications
}

\section{R. GANESAMOORTHY ${ }^{1, * \bullet}$ and P. SAKTHIVEL ${ }^{2}$}

${ }^{1}$ Department of Chemistry, School of Basic Sciences, Vels Institute of Science, Technology \& Advanced Studies (VISTAS), Chennai-600117, India ${ }^{2}$ Department of Nanoscience and Technology, Bharathiar University, Coimbatore-641046, India

*Corresponding author: E-mail: chemgmoorthy@gmail.com

Received: 7 May 2019;

Accepted: 13 June 2019;

Published online: 30 August 2019;

AJC-19547

Ten donor-acceptor based (D-A) cyano-stilbene derivatives were synthesized and their structure was confirmed by ${ }^{1} \mathrm{H}$ NMR, GC-MS, FTIR. The absorption was calculated by UV-visible spectrometer. From the UV-Vis study, stilbenes are having broad and strong absorption in the UV-visible region. The strong and broad absorption in the UV-visible region is the desired property for the high power conversion efficiency organic photovoltaic cells.

1

Keywords: Cyano-stilbene, Organic photovoltaic, Power conversion efficiency.

\section{INTRODUCTION}

The stilbene name was derived from the Greek word 'stilbos'. Stilbene derivatives can be easily prepared by some conventional and eco-friendly methods. They are thermally and chemically stable and possess absorption and fluorescence properties [1]. The stilbene are mainly present in two isomeric forms namely trans-stilbene (E) and cis-stilbene (Z). In the above two forms, trans-form is more stable than cis-form because of steric factor and high energy in the later isomer. The two isomers are in equilibrium at room temperature but at high temperature or exposed to light the trans-form is predominant because of the stability and energy difference. The energy of cis-form higher than the trans-form. They are insoluble in water but soluble in most of the common organic solvents $[1,2]$.

Stilbenes are widely used in the manufacture of industrial dyes, dye lasers, optical brighteners, phosphor materials, scintillator and other materials. Donor-acceptor (D- $\pi$-A) dyestuff and colorants represent a vast majority of organic chromogens. Some of these compounds are also used as sources of dye laser radiation $[3,4]$. They are playing an increasingly prominent role in the area of photophysical, photochemical, biophysical and biomedical investigations. Unsubstituted stilbenes are less reactive but if any hydroxyl or some other functional group present in the stilbene it is highly reactive. Stilbenoids (hydroxylated derivatives of stilbene) are produced by plants are used as an important antibiotic material in their life time. The stilbene derivatives have also medical and biological activities, such as antimicrobial, antifungal, insecticidal and anti-inflammatory $[5,6]$.

Stilbenes can be synthesized by many methods in that most important methods are given as follows, Aldol-type condensation, Wittig-Horner reaction, Perkin reaction, Negishi-Stille reactions and Heck reaction. In the above mentioned methods, Aldoltype condensation between an aromatic aldehyde with active methylarene compound is most common because of economically benign, simple and easy way of preparation in short span of time [7-11]. The Aldol condensation type of stilbene synthesis involves the formation of carbanion and addition to the carbonyl group. The strong or weak base like $\mathrm{NaOH}$, pyridine or piperidine initiates the reaction by deprotonation of active methylarene. The carbanion then adds to carbon atoms of the carbonyl group of the aldehyde [12-15].

Broad and strong absorption band in visible and near infrared region to match the solar spectrum for increasing short circuit current $\left(\mathbf{J}_{\mathrm{sc}}\right)$ [16-19]. A broad class of dyestuff and colorants are based on electron donor-acceptor (D- $\pi$-A) chromogens

This is an open access journal, and articles are distributed under the terms of the Attribution 4.0 International (CC BY 4.0) License. This license lets others distribute, remix, tweak, and build upon your work, even commercially, as long as they credit the author for the original creation. You must give appropriate credit, provide a link to the license, and indicate if changes were made. 
were synthesized by Asiri [20]. Due to the broad absorption in UV-visible region stilbenes can be used as an efficient dye for organic photovoltaic. This observation leads us to synthesis stilbene dyes for the applications in organic photovoltaic.

\section{EXPERIMENTAL}

Thiophene-2-carboxaldehyde, 4-hydroxybenzaldehyde, 4-bromobenzaldehyde, 5-bromothiophenecarboxaldehyde, ethanol, vanillin, furfural, 5-bromothiopheneacetonitrile, 4-hydroxyphenylacetonitrile, potassium tert-butoxide, 4-fluorophenylacetonitrile and 4-nitrophenylacetonitrile were purchased from Sd-Fine Chem. Ltd., India. All the solvents were distilled prior to usage.

Synthesis of cyano-stilbene: Aryl acetonitrile (20 mmol) was dissolved in $20 \mathrm{~mL}$ of ethanol and taken in a three neck flask. To this, $10 \mathrm{mmol}$ of alcoholic potassium tert-butoxide was added and stirred for $0.5 \mathrm{~h}$. An alcoholic solution of aromatic aldehyde $(20 \mathrm{mmol})$ was added dropwise into the above solution. Within a few minutes formation of coloured product was started. The reaction mixture was again stirred for $3 \mathrm{~h}$ at room temperature. Progress was monitored by TLC. The reaction mixture was then poured into crushed ice and neutralized with sufficient amount of water. The precipitated product was filtered by using a Buchner funnel. The filtered stilbene was washed with water and ethanol for several times to eliminate $\mathrm{NaOH}$ and unreacted present in the reaction mixture. The product was further purified by recrystallization in ethanol. The product formation was further confirmed by GC-MS, UVvisible, FT-IR and ${ }^{1} \mathrm{H}$ NMR. The consolidated data for the synthesized cyano-stilbenes are summarized in Table-1.

(E)-2-(5-Bromothiophen-2-yl)-3-(4-hydroxyphenyl) acrylonitrile (S-1): m.f.: $\mathrm{C}_{13} \mathrm{H}_{8} \mathrm{NOSBr}$, calculated mass: 305 , observed mass: 306. FT-IR (KBr, $v_{\max }, \mathrm{cm}^{-1}$ ): 3303 (O-H), 2945 (arom. C-H), 2270 (C-N), 1487, 1429 (arom. C=C), 615 (C$\mathrm{Br}) .{ }^{1} \mathrm{H}$ NMR $\left(400 \mathrm{MHz}, \mathrm{CDCl}_{3}\right):(\delta 7.26 \mathrm{ppm}, \mathrm{s}) 7.7(2 \mathrm{H}, \mathrm{d})$, $7.3(1 \mathrm{H}, \mathrm{d}), 7.3(1 \mathrm{H}, \mathrm{d}), 7.2(1 \mathrm{H}, \mathrm{s}), 7.0(1 \mathrm{H}, \mathrm{q}), 6.9(2 \mathrm{H}, \mathrm{d})$.

(E)-3-(4-Bromophenyl)-2-(5-bromothiophen-2yl)acrylonitrile (S-2): m.f.: $\mathrm{C}_{13} \mathrm{H}_{7} \mathrm{NSBr}_{2}$, calculated mass: 367 , observed mass: 367 . FT-IR (KBr, $v_{\max }, \mathrm{cm}^{-1}$ ): 3034 (arom. CH), 2223 (C-N), 1587, 1581, 1487 (arom. C=C), 810 (C-Br). ${ }^{1} \mathrm{H} \mathrm{NMR}\left(400 \mathrm{MHz}, \mathrm{CDCl}_{3}\right):(\delta 7.26 \mathrm{ppm}, \mathrm{s}), 7.7(2 \mathrm{H}, \mathrm{s}), 7.5$ (2H, d), $7.1(2 \mathrm{H}, \mathrm{t}), 7.0(1 \mathrm{H}, \mathrm{d})$.

(E)-2-(5-Bromothiophen-2-yl)-3-(furan-2-yl)acrylonitrile (S-3): m.f.: $\mathrm{C}_{11} \mathrm{H}_{6} \mathrm{NOSBr}$, calculated mass: 278, observed mass: 279. FT-IR (KBr, $v_{\max }, \mathrm{cm}^{-1}$ ): 2945 (arom. C-H), 2227 (arom. C-N), 1487, 1429 (arom. C=C), 695 (C-Br). ${ }^{1} \mathrm{H} \mathrm{NMR}(400 \mathrm{MHz}$, $\left.\mathrm{CDCl}_{3}\right)$ : $(\delta 7.26 \mathrm{ppm}, \mathrm{s}), 7.6(1 \mathrm{H}, \mathrm{d}), 7.1(2 \mathrm{H}, \mathrm{d}), 7.0(2 \mathrm{H}, \mathrm{t}), 6.5$ $(1 \mathrm{H}, \mathrm{q})$.

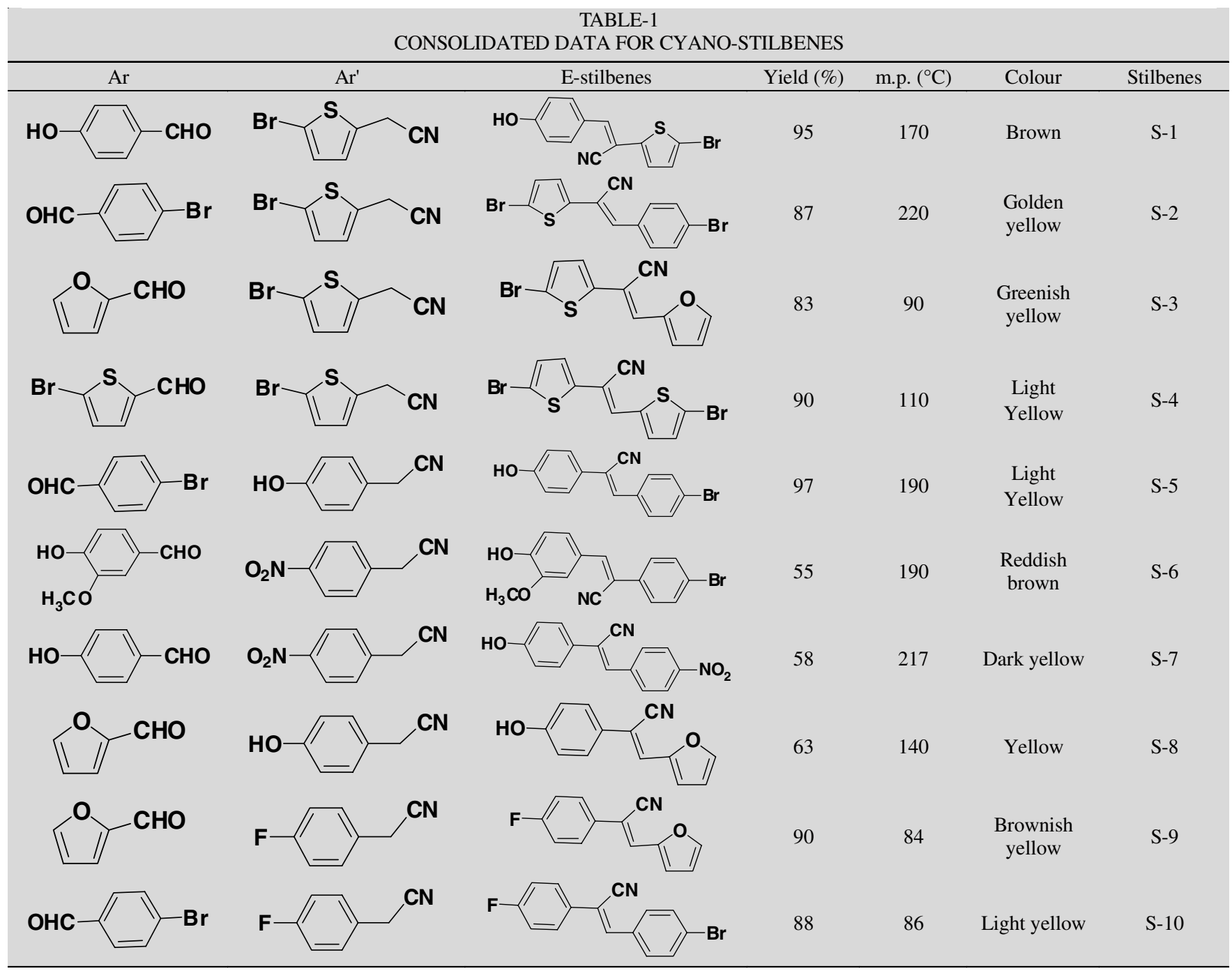


(E)-2,3-bis (5-Bromothiophen-2-yl)acrylonitrile (S-4): m.f.: $\mathrm{C}_{11} \mathrm{H}_{5} \mathrm{NS}_{2} \mathrm{Br}_{2}$, calculated mass: 372 , observed mass: 373 , FT-IR (KBr, $v_{\max }, \mathrm{cm}^{-1}$ ): 3305 (arom. C-H), 2231 (arom. C-N), $1583,1610,1514$ (arom. C=C), $821(\mathrm{C}-\mathrm{Br}) .{ }^{1} \mathrm{H} N \mathrm{MR}(400 \mathrm{MHz}$, $\left.\mathrm{CDCl}_{3}\right)$ : $(\delta 7.26 \mathrm{ppm}, \mathrm{s}), 7.27(2 \mathrm{H}, \mathrm{d}), 7.1(2 \mathrm{H}, \mathrm{t}), 7.0(1 \mathrm{H}, \mathrm{d})$.

(E)-3-(4-Bromophenyl)-2-(4-hydroxyphenyl)acrylonitrile (S-5): m.f.: $\mathrm{C}_{15} \mathrm{H}_{10} \mathrm{NOBr}$, calculated mass: 298, observed mass: 299. FT-IR (KBr, $\left.v_{\max }, \mathrm{cm}^{-1}\right)$ : 3379, $3338(\mathrm{O}-\mathrm{H}), 3230$ (arom. $\mathrm{C}-\mathrm{H}), 2227(\mathrm{C}-\mathrm{N}), 1610,1583(\mathrm{C}=\mathrm{C}), 821$ (C-Br). ${ }^{1} \mathrm{H}$ NMR $\left(400 \mathrm{MHz}, \mathrm{CDCl}_{3}\right):(\delta 7.26 \mathrm{ppm}, \mathrm{s}), 7.7(2 \mathrm{H}, \mathrm{d}), 7.5(4 \mathrm{H}, \mathrm{t}), 7.3$ $(1 \mathrm{H}, \mathrm{s}), 6.9(2 \mathrm{H}, \mathrm{d}), 5.3(1 \mathrm{H}, \mathrm{s})$.

(E)-3-(4-Hydroxy-3-methoxyphenyl)-2-(4-nitrophenyl)acrylonitrile (S-6): m.f.: $\mathrm{C}_{16} \mathrm{H}_{12} \mathrm{~N}_{2} \mathrm{O}_{4}$, calculated mass: 296, observed mass: 297. FT-IR $\left(\mathrm{KBr}, v_{\max }, \mathrm{cm}^{-1}\right): 3130(\mathrm{O}-\mathrm{H}), 3094$ (arom. C-H) 2227 (arom. C-N), $1610(\mathrm{C}-\mathrm{O}-\mathrm{C}), 1570(\mathrm{C}=\mathrm{O})$, 1215 (N-O). ${ }^{1} \mathrm{H}$ NMR $\left(400 \mathrm{MHz}, \mathrm{CDCl}_{3}\right)$ : ( $\left.87.26 \mathrm{ppm}, \mathrm{s}\right), 8.3$ $(2 \mathrm{H}, \mathrm{q}), 7.84(2 \mathrm{H}, \mathrm{q}), 7.80(1 \mathrm{H}, \mathrm{s}), 7.6(1 \mathrm{H}, \mathrm{s}), 7.3(1 \mathrm{H}, \mathrm{q}), 7.0$ $(1 \mathrm{H}, \mathrm{d}), 6.0(1 \mathrm{H}, \mathrm{s}), 4.0(2 \mathrm{H}, \mathrm{s})$.

(E)-2-(4-Hydroxyphenyl)-3-(4-nitrophenyl)acrylonitrile (S-7): $m$.f. $\mathrm{C}_{15} \mathrm{H}_{10} \mathrm{~N}_{2} \mathrm{O}_{3}$, calculated mass: 266, observed mass: 267. FT-IR $\left(\mathrm{KBr}, v_{\max }, \mathrm{cm}^{-1}\right): 3404(\mathrm{O}-\mathrm{H}), 2214(\mathrm{C}-\mathrm{N})$, 1585, (arom. $\mathrm{C}=\mathrm{C}), 1512(\mathrm{~N}=\mathrm{O}) .{ }^{1} \mathrm{H}$ NMR $\left(400 \mathrm{MHz}, \mathrm{CDCl}_{3}\right)$ : $(\delta 7.26 \mathrm{ppm}, \mathrm{s}), 8.3(2 \mathrm{H}, \mathrm{d}), 7.9(2 \mathrm{H}, \mathrm{d}), 7.8(2 \mathrm{H}, \mathrm{d}), 7.6(1 \mathrm{H}$, s), $6.9(2 \mathrm{H}, \mathrm{d})$.

(E)-3-(Furan-2-yl)-2-(4-hydroxyphenyl)acrylonitrile (S-8): m.f.: $\mathrm{C}_{16} \mathrm{H}_{12} \mathrm{~N}_{2} \mathrm{O}_{4}$, calculated mass: 296, observed mass: 297. FT-IR (KBr, $\left.\nu_{\max }, \mathrm{cm}^{-1}\right): 3269$ (arom. C-H), $2227(\mathrm{C}-\mathrm{N})$, 1614, $1591(\mathrm{C}=\mathrm{O}), 1514$ (C-Br). ${ }^{1} \mathrm{H}$ NMR $\left(400 \mathrm{MHz}, \mathrm{CDCl}_{3}\right)$ : $(\delta 7.26 \mathrm{ppm}, \mathrm{s}), 7.8(1 \mathrm{H}, \mathrm{d}), 7.6(1 \mathrm{H}, \mathrm{s}), 7.5(2 \mathrm{H}, \mathrm{d}), 7.0(1 \mathrm{H}$, d), $6.7(3 \mathrm{H}, \mathrm{m}), 5.3(1 \mathrm{H}, \mathrm{s})$.

(E)-2-(4-Fluorophenyl)-3-(furan-2-yl)acrylonitrile (S9): m.f: $\mathrm{C}_{13} \mathrm{H}_{8} \mathrm{NOF}$, calculated mass: 213, observed mass: 214. FT-IR (KBr, $\left.v_{\max }, \mathrm{cm}^{-1}\right): 3156$ (arom. C-H), 2337 (C-N), 748, 588 (C-F). ${ }^{1} \mathrm{H}$ NMR (400 MHz, $\left.\mathrm{CDCl}_{3}\right)$ : $(\delta 7.26 \mathrm{ppm}, \mathrm{s}): 7.8$ $(2 \mathrm{H}, \mathrm{d}), 7.6(\mathrm{H}, \mathrm{s}), 7.3(2 \mathrm{H}, \mathrm{d}), 7.1(2 \mathrm{H}, \mathrm{d}), 6.9(1 \mathrm{H}, \mathrm{d}), 6.4$ $(1 \mathrm{H}, \mathrm{s})$.

(E)-3-(4-Bromophenyl)-2-(4-fluorophenyl)acrylonitrile (S-10): m.f: $\mathrm{C}_{15} \mathrm{H}_{9} \mathrm{NBrF}$, calculated mass: 300 , observed mass: 302. FT-IR (KBr, $\left.\nu_{\max }, \mathrm{cm}^{-1}\right): 3026$ (arom. C-H), $2216(\mathrm{C}-\mathrm{N})$, 1834, (arom. C=C), 1512, ( C-N), 1246, (C-Br), 1078 (C-F). ${ }^{1} \mathrm{H}$ NMR $\left(400 \mathrm{MHz}, \mathrm{CDCl}_{3}\right)$ : $(\delta 7.26 \mathrm{ppm}, \mathrm{s}), 7.9(2 \mathrm{H}, \mathrm{d}), 7.8$ $(1 \mathrm{H}, \mathrm{s}), 7.6(2 \mathrm{H}, \mathrm{d}), 7.5(2 \mathrm{H}, \mathrm{d}), 7.01(2 \mathrm{H}, \mathrm{d}), 6.4(1 \mathrm{H}, \mathrm{s})$.

\section{RESULTS AND DISCUSSION}

Cyano-stilbenes are most widely used in organic solar cells to construct a D-A acceptor system with broad absorption in the UV-vis region. In this paper, we reported synthesis and characterization of some of cyano-stilbene derivatives. The UVvisible absorption studies revealed that broad and stronger absorption of cyano-stilbenes. The strong and broad absorption is the desired property for the high power conversion efficient organic photovoltaic.

The UV-vis spectra of stilbenes exhibited the presence $n$ $\pi^{*}$ and $\pi-\pi^{*}$ electronic transitions. Band around $260-300 \mathrm{~nm}$ corresponds to $\pi-\pi^{*}$ transition in $\mathrm{C}=\mathrm{C}$ (aromatic). The appearance of broad bands around $360-380 \mathrm{~nm}$ indicate to $\pi-\pi^{*}$ electronic transition of $-\mathrm{C}=\mathrm{N}$ group. In addition, the peak around 400-450 nm belongs to $n-\pi^{*}$ of $C=N$ group. 4-Fluorophenyl- acetonitrile based stilbenes such as $\mathbf{S - 9}$ and $\mathbf{S - 1 0}$ showed absorption between 250-380 $\mathrm{nm}$. Whereas, thiophene based stilbenes such as $\mathbf{S - 3 ,} \mathbf{S - 4}$ and vanillin based stilbenes such as S-6 showed maximum absorption between $250-450 \mathrm{~nm}$ (Fig. 1). The enhancement in absorption was attributed to the presence of electron rich or electron donating groups. Stilbenes like S-1, S-2, S-5, S-7 and S-8 showed medium absorption between 300-400 nm (Fig. 1). From the electronic spectra, the presence of electron rich thiophene is confirmed and furan units will enhance the absorption whereas the introduction of electron withdrawing groups such as fluoro, nitro groups will results hypsochromic shift in the absorption spectra.

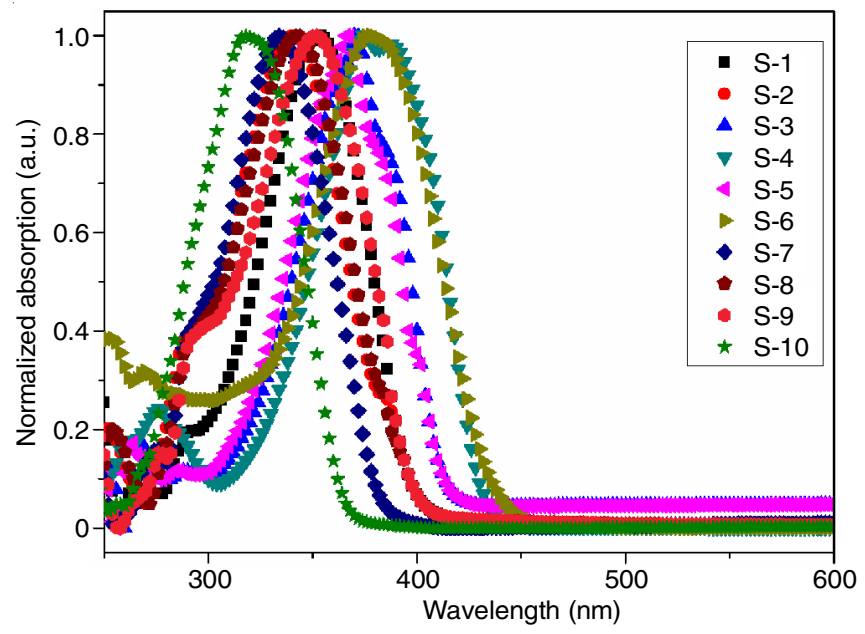

Fig. 1. UV-vis spectra of the synthesized stilbenes

\section{CONFLICT OF INTEREST}

The authors declare that there is no conflict of interests regarding the publication of this article.

\section{REFERENCES}

1. Y.-J. Cheng, S.-H. Yang and C.-S. Hsu, Chem. Rev., 109, 5868 (2009); https://doi.org/10.1021/cr900182s.

2. R. Ganesamoorthy, G. Sathiyan and P. Sakthivel, Sol. Energy Mater. Sol. Cells, 161, 102 (2017);

https://doi.org/10.1016/j.solmat.2016.11.024.

3. R. Ganesamoorthy, R. Vijayaraghavan, K. Ramki and P. Sakthivel, J. Sci.: Adv. Mater. Devices, 3, 99 (2018); https://doi.org/10.1016/j.jsamd.2017.11.005.

4. R. Ganesamoorthy, R. Vijayaraghavan and P. Sakthivel, J. Electron. Mater, 46, 6784 (2017);

https://doi.org/10.1007/s11664-017-5706-3.

5. P. Gutlich, Y. Garcia and T. Woike, Coord. Chem. Rev., 219-221, 839 (2001); https://doi.org/10.1016/S0010-8545(01)00381-2.

6. R. Grabowski, W. Rotkiewicz and W. Rettig, Chem. Rev., 103, 3899 (2003); https://doi.org/10.1021/cr9407451.

7. A. Momotake and T. Arai, J. Photochem. Photobiol. Photochem. Rev., 5, 1 (2004); https://doi.org/10.1016/j.jphotochemrev.2004.01.001.

8. F. Ververidis, E. Trantas, C. Douglas, G. Vollmer, G. Kretzschmar and N. Panopoulos, Biotechnol. J., 2, 1214 (2007); https://doi.org/10.1002/biot.200700084.

9. Y. Cai, Q. Luo, M. Sun and H. Corke, Life Sci., 74, 2157 (2004); https://doi.org/10.1016/j.lfs.2003.09.047.

10. G. Wittig and W. Haag, Chem. Ber., 88, 1654 (1955); https://doi.org/10.1002/cber.19550881110. 
11. P. Saiko, A. Szakmary, W. Jaeger and T. Szekeres, Mutat. Res., 658, 68 (2008); https://doi.org/10.1016/j.mrrev.2007.08.004.

12. M. Lin and C.S. Yao, Stud. Nat. Prod. Chem., 33, 601 (2006); https://doi.org/10.1016/S1572-5995(06)80035-9.

13. A. Cravino, H. Neugebauer, A. Petr, P.J. Skabara, H.J. Spencer, J.W. McDouall, L. Dunsch and N.S. Sariciftci, J. Phys. Chem. B, 110, 2662 (2006); https://doi.org/10.1021/jp0562173.

14. H.J. Spencer, P.J. Skabara, M. Giles, I. McCulloch, S.J. Coles and M.B. Hursthouse, J. Mater. Chem., 15, 4783 (2005); https://doi.org/10.1039/b511075k.

15. R. Ganesamoorthy, R. Vidya and P. Sakthivel, IET Nanobiotechnol., 12, 147 (2018);

https://doi.org/10.1049/iet-nbt.2017.0033.
16. Y. Li, Acc. Chem. Res., 45, 723 (2012); https://doi.org/10.1021/ar2002446.

17. J.H. Kwon, J.Y. An, H. Jang, S. Choi, D.S. Chung, M.J. Lee, H.J. Cha, J.H. Park, C.E. Park and Y.H. Kim, J. Polym. Sci. A, 49, 1119 (2011); https://doi.org/10.1002/pola.24526.

18. K.H. Kim, H. Kang, S.Y. Nam, J. Jung, P.S. Kim, C.H. Cho, C. Lee, S.C. Yoon and B. Kim, Chem. Mater, 23, 5090 (2011); https://doi.org/10.1021/cm202885s

19. Y. Zou, B. Peng, B. Liu, Y. Li, Y. He, K. Zhou and C. Pan, J. Appl. Polym. Sci., 115, 1480 (2010); https://doi.org/10.1002/app.31080.

20. A.M. Asiri, Bull. Korean Chem. Soc., 24, 426 (2003); https://doi.org/10.5012/bkcs.2003.24.4.426. 